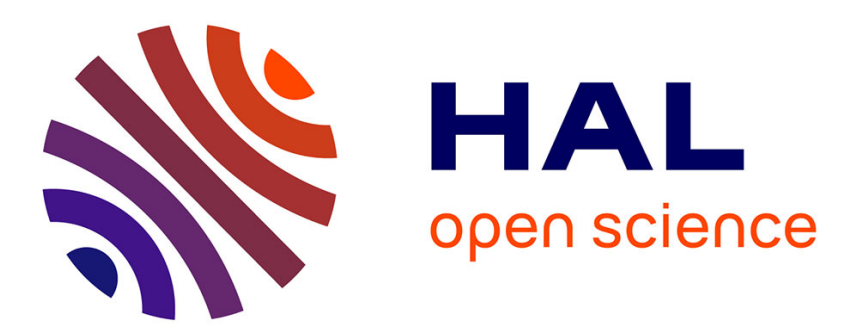

\title{
Binding kinetics calculations of the inclusion of some benzoic acid derivatives and paracetamol in $\beta \mathrm{CD}$
}

\author{
Djamel Eddine Khatmi, Belgacem Bezzina, Stéphane Humbel
}

\section{To cite this version:}

Djamel Eddine Khatmi, Belgacem Bezzina, Stéphane Humbel. Binding kinetics calculations of the inclusion of some benzoic acid derivatives and paracetamol in $\beta$ CD. Journal of Inclusion Phenomena and Macrocyclic Chemistry, 2020, 96 (3-4), pp.373-379. 10.1007/s10847-020-00976-1 . hal-02922032

\section{HAL Id: hal-02922032 \\ https://hal.science/hal-02922032}

Submitted on 25 Aug 2020

HAL is a multi-disciplinary open access archive for the deposit and dissemination of scientific research documents, whether they are published or not. The documents may come from teaching and research institutions in France or abroad, or from public or private research centers.
L'archive ouverte pluridisciplinaire HAL, est destinée au dépôt et à la diffusion de documents scientifiques de niveau recherche, publiés ou non, émanant des établissements d'enseignement et de recherche français ou étrangers, des laboratoires publics ou privés. 
Binding kinetics calculations of the inclusion of some benzoic acid derivatives and paracetamol in $\beta \mathrm{CD}$.

Djamel Eddine Khatmi,${ }^{\mathrm{a}, \mathrm{b}^{*}}$ Belgacem Bezzina, ${ }^{\mathrm{b}, \mathrm{c}}$ Stéphane Humbel, ${ }^{\mathrm{a}}$

a- Aix Marseille University, CNRS, Centrale Marseille, iSm2, Marseille, France

${ }^{\text {b- }}$ Laboratory of Computational Chemistry and Nanostructures, University of 08 May 45 Guelma, Algeria

${ }^{c-}$ Research Center in Industrial Technology (CRTI), P.O.BOX 64, Chéraga 16014, Algiers, Algeria

*corresponding author: khatmi.djameleddine@gmail.com

KEYWORDS: Cyclodextrin. Inclusion complexes. Molecular dynamic simulation. Binding Kinetics. Benzoic acid derivatives. Paracetamol. 


\begin{abstract}
This theoretical study is designed to identify the main driving forces of the inclusion of some benzoic acid (BA) derivatives as well as Paracetamol (ParCM) with $\beta$-cyclodextrin $(\beta-\mathrm{CD})$ by computing the kinetics of binding. Unbiased molecular dynamics simulations were carried out. The guest molecules were used in ionized and non-ionized states. Based on the computed values of the dissociation rate constant $k_{o f f}$, the hydrophobic interactions were discussed. The results show that the more hydrophobic molecule in the non-ionized state give most stable complexes; whereas in the ionized state, less hydrophobic molecules give most stable complexes, meaning a clear charge effect. The low percentage of time of observed H-bonds during the simulations is an indication that ones may play only a secondary role in the stability of the non-ionized inclusion complexes, and they don't play any role in the stability of the ionized complexes. The comparison between the obtained results and those obtained experimentally gives a reasonably agreement.
\end{abstract}




\section{INTRODUCTION}

Cyclodextrin is an excellent model for the molecular recognition because of its structure, with a hydrophobic cavity, may include various small molecules. The guest molecules' chemical properties such as solubility, stability and biological activity can change due to its complexation with a cyclodextrin. This technique of encapsulation has become very popular and is being successfully used in different fields, chemistry, biology, nano chemistry, agro chemistry and industry [1-5].

The ease of the inclusion depends on various factors in particular the hydrophobicity and hydrogen bonding [6-8]. The ionized state of the guest compound has also a significant effect on the complexation and can considerably affect the stability of the inclusion complexes (ICs) [9-11].

The molecular binding of the ICs can be characterized through thermodynamic or kinetic properties. Thus, the thermodynamic properties are usually obtained by determining the energy profile of the complexation using biased molecular dynamic simulation method and the kinetic properties by unbiased molecular dynamics simulation method. These methods are becoming increasingly prominent for studying molecular binding as well as for determining the efficiency of a drug [12-13].

In this article we present the computed results on the kinetics of binding of the inclusion of some BA derivatives as well as ParCM in order to give a better understanding of the complexation and its binding mechanism. For this purpose, we computed the kinetic properties using unbiased molecular dynamic simulations. The choice of BA derivatives as guest molecules is justified by the fact that they have suitable structural features to be encapsulated in $\beta-C D$, and also by the number of the experimental results related to their ICs $[6,9-12,14-19]$. To note that ParCM is a more hydrophilic compound, and was also used as guest molecule in order to compare its kinetic behavior to those of BA derivatives. 


\section{METHODS}

The initial structure of $\beta-C D$ was built with the help of the graphical interface of ChemBioOffice (version 10, Cambridge software) [20], and the geometry of guest molecules was extracted from a PubChem Compound Database [21] (Figure 1).

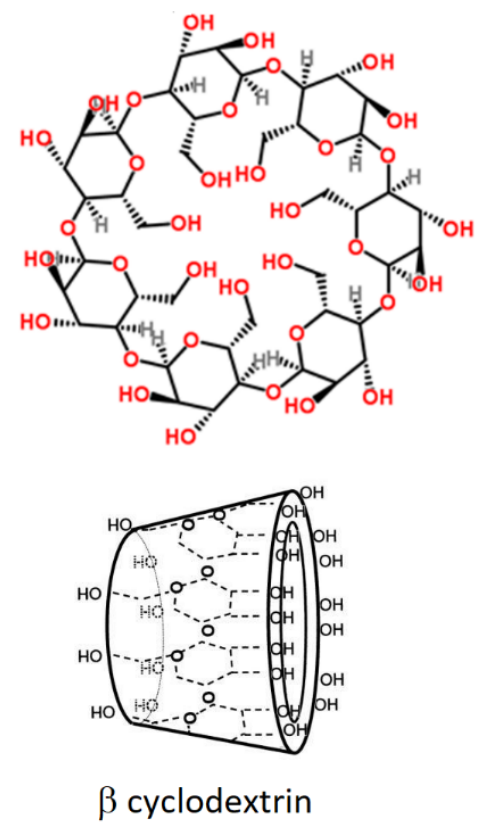

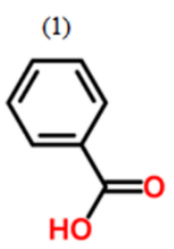

(4)<smiles>Cc1ccc(C(=O)O)cc1</smiles><smiles>CC(=O)Oc1ccccc1C(=O)O</smiles>

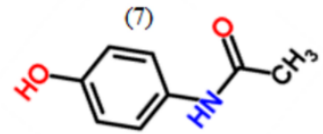<smiles>O=C(O)c1ccccc1O</smiles><smiles>[CH2]C([CH2+])Cc1ccc(C([18O])C(=O)O)cc1</smiles>

Figure 1. $\beta-\mathrm{CD}$ and the 7 guest molecular structures, (1) benzoic acid (BA), (2) toluic acid (TA), (3) salicylic acid (SA), (4) phenyl acetic acid (PAA), (5) aspirin (ASP), (6) ibuprofen (IBU) and (7) paracetamol (ParCM)

First, $\beta-\mathrm{CD}$ is fixed in the center of $\mathrm{XYZ}$ coordinate system. Then, the ionized or non-ionized guest molecule is placed to a distance greater than $10 \AA$ from the center of $\beta-C D$ (Figure 1 ).

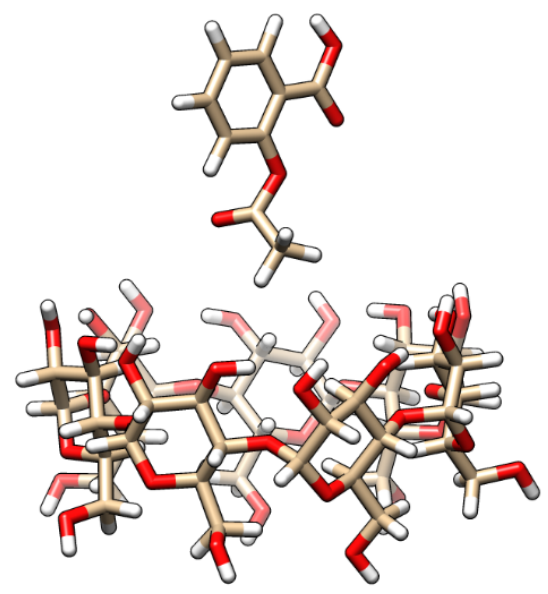


Figure 2. The initial geometry at the beginning of the simulation (ionized ASP with $\beta-C D$ ).

The force field parameters for both molecules were generated using the automated topology builder (ATB) [22] in the framework of GROMOS96 54A7 force field [23].

All simulations were carried out using the GROMACS package version 5.1.2 into an identical box containing 2380 water molecules represented by a simple point charge (SPC) model [24, 25]. The simulations were performed in the isobaric-isothermal ensemble (NPT) with a constant pressure of 1 bar and a temperature of $298 \mathrm{~K}$ controlled by the Berendsen thermostat. The electrostatic interactions were treated by the particle mesh Ewald (PME) method with a cutoff distance of $1.2 \mathrm{~nm}$ and the van der Waals interactions were modeled using a cutoff of $1.4 \mathrm{~nm}$. The whole system was relaxed using the steepest descent algorithm. The position of the guest molecule is obviously altered after the equilibration steps. The classical Newton's equations of motion were integrated using the Leap-Frog algorithm and the Berendsen weakcoupling method. All simulations were carried out for $200 \mathrm{~ns}$, which is long enough for the system to reach equilibrium. The trajectory coordinates were recorded every 500 steps with a time step of 1 fs. The lifetime inside $\beta-\mathrm{CD}$ cavity is quantified by inspecting the distance between the centroîd of $\beta-C D$ and that of guest molecule during the simulations.

To ensure that we have a correct canonical ensembles as well as correct structural and thermodynamic properties we used in a second step, the Langevin thermostat in addition of a more sophistical water model, TIP3P.

The inclusion complex can be represented as a simple two-state model for guest reaction with $\beta-\mathrm{CD}$.

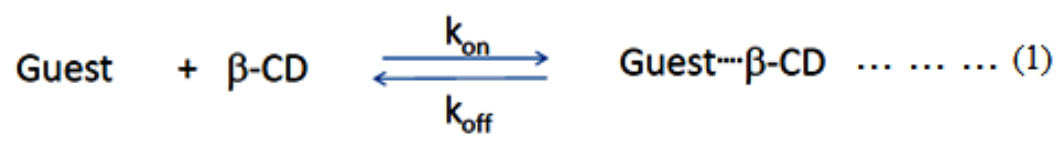

The kinetic are assumed to be first-order with the association and dissociation rate constants $k_{\text {on }}$ and $k_{\text {off }}$ which can be evaluated as follows:

$$
k_{\text {on }}=\frac{1}{\text { average unbound timeX }[\text { solute }]} \ldots \ldots \ldots
$$

and,

$$
k_{\text {off }}=\frac{1}{\text { average bound time }} \cdots \cdots \cdots
$$


The thermodynamic and kinetic behaviors are related by the equation:

$$
K=\frac{k_{o n}}{k_{o f f}} \cdots \cdots \cdots(4)
$$

The binding free energy can be obtained from the constant $K$ that:

$$
\Delta G^{\circ}=-R T \ln K
$$

\section{RESULTS AND DISCUSSION}

The values of the association and dissociation constant rate $k_{o n}$ and $k_{o f f}$, the equilibrium constant $\mathrm{K}$ and the binding standard free energy $\Delta \mathrm{G}^{\circ}$ of the ICs (ionized and non-ionized) were calculated using eq. 2, 3, 4 and 5. The results are shown in Table 1 and Table 2. Also, these tables include data extracted from the literature, obtained either by experimental methods or by molecular dynamics calculations. Note that all values in brackets correspond to those obtained for the simulations under the conditions of a TIP3P water model and a Langevin thermostat.

The more negative free standard energy gives the more thermodynamically favorable inclusion complex. The results summarized in Table 1 and 2 indicate clearly that all the molecules used, in both states, can form stable ICs with $\beta-\operatorname{CD}\left(\Delta G^{\circ}<0\right)$.

\section{Non-ionized state}

Firstly, the MD simulations of the non-ionized ICs using SPC water model and the Berendsen thermostat were divided into three groups according to the guest molecule behavior during the simulation. The first group involves four BA derivatives: PAA, BA, ASP and IBU. These guest molecules once they penetrated inside the cavity, will stay there until the end of the simulation. The obtained complexes are stable giving the same computed values of the kinetics and thermodynamics parameters. Thus, $\Delta G^{\circ}$ and $K$ are respectively equal to -4.05 $\mathrm{kcal} / \mathrm{mol}$ and $9.010^{2}$ corresponding to a dissociation constant rate value, $k_{\text {off }}$, equal to 5.26 $10^{6} \mathrm{~s}^{-1}$. To note, that $k_{\text {off }}$ values are the lowest ones, this is an indication that the hydrophobicity is larger. Accordingly, the guest molecules with a larger hydrophobicity give the more stable complexes.

Table 1. The rate association and dissociation constants $k_{o n}$ and $k_{\text {off }}$ and thermodynamic parameters $K, p k_{a}$ and $\Delta G^{\circ}$ of the ICs in the non ionized states. 


\begin{tabular}{|l|c|c|c|c|c|}
\hline & $\begin{array}{c}k_{\text {off }}\left(s^{-1}\right) \\
10^{6}\end{array}$ & $\begin{array}{c}k_{\text {on }}\left(s^{-1} M^{-1}\right. \\
10^{9}\end{array}$ & $\begin{array}{c}K\left(M^{-1}\right) \\
10^{2}\end{array}$ & $\begin{array}{c}\Delta \mathrm{G}^{\circ} \\
\text { This study }\end{array}$ & $\begin{array}{c}\Delta \mathrm{G}^{\circ} \\
\text { From literature }\end{array}$ \\
\hline SA & $5.43[5.27]$ & $2.97[4.65]$ & $5.47[8.62]$ & $-3.76[-4.03]$ & $\begin{array}{c}-3.59^{(9)} ;-2.85^{(15)} \\
-3.96^{(6)}\end{array}$ \\
\hline TA & $5.40[5.19]$ & $3.16[6.32]$ & $5.85[12.18]$ & $-3.79[-4.23]$ & $\mathrm{N} / \mathrm{A}$ \\
\hline PAA & $5.26[5.18]$ & $4.74[6.78]$ & $9.00[13.09]$ & $-4.05[-4.27]$ & $-2.82^{(9)}$ \\
\hline BA & $5.26[5.39]$ & $4.74[3.22]$ & $9.00[5.97]$ & $-4.05[-3.81]$ & $\begin{array}{c}-3.77^{(9)} ;-3.6^{(18)} \\
-2.79^{(14)}\end{array}$ \\
\hline ASP & $5.26[5.32]$ & $4.74[3.95]$ & $9.00[7.42]$ & $-4.05[-3.94]$ & $\begin{array}{c}-3.74^{(10)} ;-3.19^{(9)} ; \\
-2.98^{(9)} ;-2.27^{(12)} \\
\text { GAFF6-CD; }\end{array}$ \\
& & & & & \\
& & & & & $-4.11^{(12)}, \mathrm{q} 4 \mathrm{MD}-\mathrm{CD}$ \\
\hline IBU & $5.26[5.15]$ & $4.74[8.01]$ & $9.00[15.55]$ & $-4.05[-4.38]$ & $-4.28^{(9)} ;-6.45^{(15)}$ \\
\hline ParCM & $6.00[5.82]$ & $1.35[2.90]$ & $2.25[2.90]$ & $-3.2[-3.37]$ & $-2.98^{(16)}$ \\
\hline
\end{tabular}

The second group consists of SA and TA. During the simulation, these molecules are easily encapsulated inside the cavity staying until the end of the simulation. But sometimes, the guest molecules leave the cavity for short periods. A small difference is observed between the values of the free energies $\Delta G^{\circ}$ of both complexes as well as between the values of the equilibrium constants $K$. The values of $\Delta G^{\circ}$ are equal to -3.76 (SA- $\left.\beta-\mathrm{CD}\right)$ and -3.79 (TA- $\beta$ CD) $\mathrm{kcal} / \mathrm{mol}$ and the values of $K$ are equal to $5.4710^{2}$ (SA- $\left.\beta-\mathrm{CD}\right)$ and $5.8510^{2}$ (TA- $\left.\beta-\mathrm{CD}\right)$.

However, the values of $k_{\text {off }}$ reported in Table 1 shows a higher values, equal to $5.4310^{6}$ (SA$\beta-\mathrm{CD}$ ) and $5.4010^{6}$ (TA- $\left.\beta-\mathrm{CD}\right)$ compared to those obtained in the first group unveils to a lower hydrophobicity, leading in consequence to a slightly less stable ICs.

Finally, the last group is composed of only one molecule, ParCM. In this case, ParCM gives also a complex with $\beta-\mathrm{CD}$ but sometimes leaves the cavity, staying much longer periods outside compared to the guest molecules of the second group. The computed value of $k_{o f f}$ is much higher and equal to $6.010^{2} \mathrm{~s}^{-1}$ indicating that ParCM is the least hydrophobic. This leads to the least stable IC characterized by a free energy $\Delta G^{\circ}$ of $-3.2 \mathrm{kcal} / \mathrm{mol}$ and a equilibrium constant of $2.2510^{2}$.

However a second time, when using the Langevin thermostat and the TIP3P water model, the behavior of the non-ionized ICs is becoming slightly different for TA and SA; this time once they have penetrated inside the cavity, they do not leave it anymore unlike previous simulations where they came out from time to time. In other words all benzoic acids, PAA, 
BA, ASP, IBU, SA and TA behave the same way in these conditions. However, in the ParCM: $\beta-C D$ complex, the ParCM behaves the same way, i.e. it go in and out the cavity several times during the MD simulation.

Comparing the values of $\Delta \mathrm{G}^{\circ}$ in the first and second MD simulations, we observe an average difference of $0.25 \mathrm{kcal} / \mathrm{mol}$ corresponding certainly of the necessary fitting obtained by using the Langevin thermosat and the TIP3P water model, to make the results more accurate.

\section{The ionized state}

In this section we describe and discuss the MD simulations of the ICs of five BA derivatives in their ionized state TA, SA, PAA, BA and ASP with $\beta-C D$.

Table 2. The rate association and dissociation constants $k_{o n}$ and $k_{\text {off }}$ and thermodynamic parameters $K, p k_{a}$ and $\Delta G^{\circ}$ of the ICs in the ionized states.

\begin{tabular}{|c|c|c|c|c|c|}
\hline & $\begin{array}{c}k_{\text {off }}\left(s^{-1}\right) \\
10^{6}\end{array}$ & $\begin{array}{c}k_{\text {on }}\left(s^{-1} M^{-1}\right) \\
10^{9}\end{array}$ & $\begin{array}{c}K\left(M^{-1}\right) \\
10^{2}\end{array}$ & $\begin{array}{c}\Delta \mathrm{G}^{\circ} \\
\text { This study }\end{array}$ & $\begin{array}{c}\Delta \mathrm{G}^{\circ} \\
\text { From literature }\end{array}$ \\
\hline SA & $6.71[6.9]$ & $0.93[0.86]$ & $1.38[1.24]$ & $-2.93[-2.86]$ & $-2.77^{(9)} ;-2.68^{(6)}$ \\
\hline TA & $5.71[5.64]$ & $1.90[2.06]$ & $3.32[3.65]$ & $-3.45[-3.51]$ & N/A \\
\hline PAA & $7.76[6.22]$ & $0.67[1.21]$ & $0.86[1.95]$ & $-2.65[-3.14]$ & N/A \\
\hline BA & $10.41[8.1]$ & $0.46[0.61]$ & $0.44[0.75]$ & $-2.25[-2.53]$ & $-1.4^{(18)} ;-2.37^{(9)}$ \\
\hline ASP & $12.82[8.6]$ & $0.39[0.56]$ & $0.30[0.56]$ & $-2.02[-2.39]$ & $-2.34^{(10)}$ \\
\hline
\end{tabular}

As it was expected, the equilibrium constant decreased significantly in the ICs of PAA, BA and ASP compared to the values of their corresponding non-ionized ICs; it is approximately 17, 21 and 31 times smaller respectively, while they are only 1.5 and 4 times smaller for TA and SA respectively.

Finally, it seemed interesting to establish a relationship between the hydrophobicity and the stability of the ionized ICs based on the values of $k_{\text {off }}$ and $K$. Thus, from Table 1 the computed values $K$ can be ordered according to: $K_{A S P}<K_{B A}<K_{P A A}<K_{S A}<K_{T A}$ and the computed values $k_{o f f}$ according to: $k_{o f f_{A S P}}>k_{o f f_{B A}}>k_{o f f_{P A A}}>k_{o f f_{S A}}>k_{o f f_{T A}}$. This demonstrates that the less hydrophobic guest molecule gives the more stable complex on the contrary of the general rule of the formation of the ICs and with respect to our results obtained in the non-ionized state. This means that a clear charge effect in the guest molecules probably makes them less hydrophobic, and this is reflected in the process of their separation from the $\beta$-CD. Consequently, we can admit that the hydrophobic interaction is not the most 
determining factor in the formation of these ICs, instead, the effect of charge which becomes the most important factor.

When using the Langevin thermostat and TIP3P water model in the simulations the behavior of the ionic ICs did not change, i.e. the invited molecules leaves the cavity several times during the simulation; deducing then the same relationship between the hydrophobicity and the stability of the ionized ICs based on the values of $k_{o f f}$ and $K: K_{A S P}<K_{B A}<K_{P A A}<K_{S A}<$

$K_{T A}$ and the computed values $k_{o f f}$ according to: $k_{o f f_{A S P}}>k_{o f f_{B A}}>k_{o f f_{P A A}}>k_{o f f}>k_{\text {off }}{ }_{T A}$.

It should be noted that although, we observe an average difference of $0.25 \mathrm{kcal} / \mathrm{mol}$ in the calculated values of $\Delta \mathrm{G}^{\circ}$ between the two simulations, this has not affected the behavior of the guest molecules in this case.

The experimental values listed in Table 1 and Table 2 have been obtained using different technic, the potentiometric measurement [6], the simultaneous measurement of absorbance and circular dichroism [9], the ultrasonic relaxation [10], the low frequency ultrasonic relaxation [14] thermodynamics [15], UV-Visible [16] and the microcalorimetry [18]. Also two theoretical results obtained by Chang et al are cited, they use two different forces fields for MD simulation of Asp: $\beta-C D(12)$. As it can be seen the data listed are scattered. They are, of because of the methods used using are different, or by the fact that these experiments were conducted in different measurement environments $(\mathrm{pH}, \mathrm{T}$, solvent). In addition, they are not complete and do not concern all of our examples. Hence, it is not straightforward to compare our results to those obtained experimentally in a fair and informative manner.

Taking into account these facts we can say that our results are overall more or less in line with those obtained experimentally in particular those obtained by Dehab et al [9] except in the ASP: $\beta-C D$ and PAA: $\beta-C D$ complexes which the difference in the $\Delta G^{\circ}$ values was found slightly significant, equal respectively to $0.86[0,75]$ and $1.23[1.45] \mathrm{kcal} / \mathrm{mol}$.

It also seemed to us that, despite its well-known limitations, using the Berendsen thermostat and the SPC water model in MD simulation may provide with a good approximation a satisfactory results, as shown the results on Table 1 and Table 2.

\section{Hydrogen bonding}

Here, we shall focus our main attention on the formation of the H-bonds between $\beta$-CD and guest molecules in both states throughout the simulation [26]. A hydrogen bond is considered 
as formed during the simulation when the donor-acceptor distance is less than or equal to 3.0 $\AA$ and an angle between donor-hydrogen-acceptor is greater than or equal to $120^{\circ}$.

In Table 2 are displayed the distribution of the number of $\mathrm{H}$-bonds observed between the guest molecules in both states and $\beta \mathrm{CD}$ during the simulation period.

Table 2. The distribution of the number of $\mathrm{H}$ bonds observed during the simulation

\begin{tabular}{|l|l|l|l|}
\hline \multicolumn{2}{|c|}{} & \multicolumn{2}{l|}{ Percentage of time (\%) } \\
\cline { 3 - 4 } \multicolumn{2}{|c|}{ Number of H-bond } \\
\hline Compound & State & 0 & 1 \\
\hline BA & non-ionized & $96.5[94]$ & $3.5[4]$ \\
\hline & Ionized & $96[92]$ & $3[7]$ \\
\hline TA & non-ionized & $87[94.5]$ & $20.5[3]$ \\
\hline & Ionized & $79[83]$ & $12[15]$ \\
\hline SA & non-ionized & $88[91]$ & $11.5[7]$ \\
\hline & Ionized & $88[77]$ & $11[19]$ \\
\hline PAA & non-ionized & $92[86]$ & $8[10]$ \\
\hline & ionized & $88[81]$ & $12[17]$ \\
\hline ASP & non-ionized & $78[72]$ & $20[25]$ \\
\hline & ionized & $98.5[95]$ & $1.5[3]$ \\
\hline IBU & non-ionized & $88[92]$ & $11[5]$ \\
\hline ParCM & non-ionized & $69[61]$ & $30[35]$ \\
\hline
\end{tabular}

The distribution (in \%) of the number of $\mathrm{H}$ bonds observed during the simulation given in Table 2, provides a general trend of non-affinity to the establishment of H-bonds except with ParCM which gives a moderate rate, about 30\% [35\%]. In other words, during about 69 [61 $\%$ ] to $98 \%$ [95\%] of the time of simulations the guest molecules do not establish any hydrogen bond with $\beta$ CD. Thus, in the two states of the ICs of BA and SA, the same distribution of one H-bond were observed; they are around of 3\% [4\%] for the $\beta$-CD-BA complex and of $11 \%$ [7\%]for the $\beta$-CD-SA complex. However, slightly different rates are detected in the ICs of PAA and TA in which the non-ionized complexes exhibit a larger proportion of one H-bond than in the ionized complexes of $4 \%$ [7\%] ( $\beta$-CD-PAA complex) and of $7.5 \%$ [12\%] ( $\beta$-CD-TA complex). This difference is more pronounced in the case of $\beta$-CD-ASP complex where the non-ionized complex gives a percentage of $20 \%$ [25\%] while the ionized state gives only $1.5 \%$ [3 \%]. Lastly, IBU give $11 \%$ [5 \%]of one H-bonds.

It is obvious that the small rate of the ionized ICs, can be explained by the fact that the ionized molecule spent much more time in aqueous medium away from the $\beta$-CD leading to less opportunity for establishing H-bonds. However, the hydrophobic interactions in the nonionized ICs are more significant in the complexation; we suppose that likely the systems don't 
need much more additional favorable interactions for their stabilities. In the non-ionized ICs, guest molecules establish more than one $\mathrm{H}$-bond with $\beta-\mathrm{CD}$; because of their freely moving inside the cavity, these H-bonds are formed and broken quickly giving them an intermittent and weakness character. Hence, it seems that H-bonds play only a secondary role in the nonionized ICs.

Finally, note that a second hydrogen bond is significantly less detectable and its distribution would not exceed $2 \%$ in the best cases.

\section{CONCLUSION}

The kinetics of the association/dissociation inclusion process of some benzoic acid derivatives and paracetamol in the $\beta \mathrm{CD}$ was investigated using unbiased molecular dynamic simulations. The results show clearly a charge effect in the inclusion process of the ionized guest molecules which reduce considerably the stability of their corresponding inclusion complexes. We conclude that less hydrophobic compounds gives the more stable complex on contrary to the non-ionized guest molecules in which the more hydrophobic compound gives the more stable complex, and also H-bond interactions play only a secondary role in the stability of the non-ionized complexes, and do not play any role in the stability of the ionized complexes. 


\section{REFERENCES}

1. Szente, Lajos, and Jozsef Szejtli. "Cyclodextrins as food ingredients." Trends in Food Science \& Technology 15.3-4 (2004): 137-142.

2. Szente, Lajos, and Julianna Szemán. "Cyclodextrins in analytical chemistry: hostguest type molecular recognition." (2013): 8024-8030.

3. Saenger, Wolfram. "Cyclodextrin inclusion compounds in research and industry." Angewandte Chemie International Edition in English 19.5 (1980): 344-362.

4. Dodziuk, Helena, ed. Cyclodextrins and their complexes: chemistry, analytical methods, applications. John Wiley \& Sons, 2006.

5. Frömming, Karl-Heinz, and József Szejtli. Cyclodextrins in pharmacy. Vol. 5. Springer Science \& Business Media, 1993.

6. Junquera, Elena, David Ruiz, and Emilio Aicart. "Role of hydrophobic effect on the noncovalent interactions between salicylic acid and a series of $\beta$ cyclodextrins." Journal of colloid and interface science 216.1 (1999): 154-160.

7. Liu, Lei, and Qing-Xiang Guo. "Use of quantum chemical methods to study cyclodextrin chemistry." Journal of inclusion phenomena and macrocyclic chemistry 50.1-2 (2004): 95-103.

8. Matsui, Yoshihisa, Takaaki Nishioka, and Toshio Fujita. "Quantitative structurereactivity analysis of the inclusion mechanism by cyclodextrins." Biomimetic and Bioorganic Chemistry. Springer, Berlin, Heidelberg, 1985. 61-89.

9. Dahab, Ali Aboel, and Dhia El-Hag. "Rapid analysis of NSAIDs binding to $\beta$ cyclodextrin using the simultaneous measurement of absorption and circular dichroism with a novel multi-cell low-volume device." Analytical and bioanalytical chemistry 404.6-7 (2012): 1839-1850.

10. Fukahori, Takanori, Minako Kondo, and Sadakatsu Nishikawa. "Dynamic study of interaction between $\beta$-cyclodextrin and aspirin by the ultrasonic relaxation method." The Journal of Physical Chemistry B 110.9 (2006): 4487-4491.

11. Loftsson, Thorsteinn, et al. "Cyclodextrin complexation of NSAIDSs: physicochemical characteristics." European Journal of Pharmaceutical Sciences 1.2 (1993): 95-101.

12. Tang, Zhiye, and Chia-en A. Chang. "Binding thermodynamics and kinetics calculations using chemical host and guest: a comprehensive picture of molecular recognition." Journal of chemical theory and computation 14.1 (2017): 303-318. 
13. Cova, Tânia FGG, et al. "Drastic stabilization of junction nodes in supramolecular structures based on host-guest complexes." Macromolecules 51.7 (2018): 2732-2741.

14. S, Park, J R Bae. "Ultrasonic Relaxation Study of the Interaction of $\beta$-Cyclodextrin with Benzoic Acid in an Aqueous Solution.” New Phys: Sae Mulli 68 (2018), 496500.

15. Castronuovo, Giuseppina, and Marcella Niccoli. "Thermodynamics of inclusion complexes of natural and modified cyclodextrins with acetylsalicylic acid and ibuprofen in aqueous solution at 298 K." Thermochimica acta 557 (2013): 44-49.

16. El-Kemary, Maged, et al. "Inclusion of Paracetamol into $\beta$-cyclodextrin nanocavities in solution and in the solid state." Spectrochimica Acta Part A: Molecular and Biomolecular Spectroscopy 79.5 (2011): 1904-1908.

17. Song, Le Xin, et al. "Formation, structure, and stability of $\alpha$-and $\beta$-cyclodextrin inclusion complexes of phenol and benzoic acid derivatives in vacuo and in water." Bulletin of the Chemical Society of Japan 80.12 (2007): 2313-2322.

18. Siimer, E., M. Kurvits, and A. Köstner. "Thermochemical investigation of $\beta$ cyclodextrin complexes with benzoic acid and sodium benzoate." Thermochimica acta 116 (1987): 249-256.

19. Jong-Rim, Bae. "Low-frequency Ultrasonic Relaxation of $\beta$-Cyclodextrin and Aspirin in Aqueous Solution”. New Physics: Sae Mulli, 65.1 (2015) 90-95.

20. Kerwin, Sean M. "ChemBioOffice ultra 2010 suite." (2010): 2466-2467.

21. Bolton, Evan E., et al. "PubChem: integrated platform of small molecules and biological activities." Annual reports in computational chemistry. Vol. 4. Elsevier, 2008. 217-241.

22. Malde, Alpeshkumar K., et al. "An automated force field topology builder (ATB) and repository: version 1.0." Journal of chemical theory and computation 7.12 (2011): 4026-4037.

23. Schmid, Nathan, et al. "Definition and testing of the GROMOS force-field versions 54A7 and 54B7." European biophysics journal 40.7 (2011): 843.

24. Abraham, Mark James, et al. "GROMACS: High performance molecular simulations through multi-level parallelism from laptops to supercomputers." SoftwareX 1 (2015): $19-25$.

25. Van Der Spoel, David, et al. "GROMACS: fast, flexible, and free." Journal of computational chemistry 26.16 (2005): 1701-1718. 
26. Alagona, Giuliano, Caterina Ghio, and Susanna Monti. "Structure and dynamics of the hydrogen-bond network around ( $\mathrm{R}, \mathrm{R})$-pterocarpans with biological activity in aqueous solution." The Journal of Physical Chemistry B 109.35 (2005): 16918-16925. 